\title{
Cognitive Enhancement Techniques and Their Impact on Performance Improvements: A Review
}

\author{
Fares Al-Shargie, C.M.GOH, Hasan Al-Nashash
}

Department of Electrical Engineering, American University of Sharjah, UAE

\begin{abstract}
The attempts to enhance the healthy cognitive function with brain disorders for patients has been an important research topic in recent years due to its importance in improving the cognitive operations of the brain, e.g. Improved learning and focusing. However, existing techniques are less robust due to the substantial variability within individuals as well as the response across. Hence, this paper provides a comprehensive survey of cognitive enhancement techniques and sustain attention. Besides, the study also discussed the biological factors that might involve such variability.
\end{abstract}

INDEX TERMS Binaural Beats, Biofeedback, Caffeine, Cognition, Meditation, Mental Training, Music. Sports and Exercise, and Transcranial direct current simulation.

\section{INTRODUCTION}

Cognitive enhancement is the amplification of the mental processes through improvement or augmentation of information processing systems. The term "cognitive enhancement" usually characterized by human interventions which aim to improve the essential operation of mental for sustaining or restoring good health. Such intervention is employed to enhance the cognitive functioning of brains such as the learning improvement, the focus enhancement, the attentional control, the memory enhancement, the faster reaction time, the better perception and the reasoning capacity improvement. Most efforts to enhance cognition are rather mundane, and have long history of practiced. Education and training are one of the examples, where the goal is to impart specific skills or information and to improve general mental faculties such as concentration, memory, and critical thinking. Besides that, the other forms of basic mental training are yoga, martial arts, meditation, and creativity courses.

\section{COGNIIVE ENHANCEMENT TECHNIQUES}

\section{A. BINAURAL BEATS}

The effects of binaural beats on cognitive enhancement have recently introduced. Binaural beats are perceived by the listener when two auditory stimuli of different frequency are presented to each ear, while the binaural beat frequency is equal to the difference between the frequencies applied to each ear. For example, when a $124 \mathrm{Hertz}(\mathrm{Hz})$ tone is sent to the left ear and a 114 Hertz tone in the right, this will yield a beat with a frequency of 10 Hertz. All tones must be presented in a frequency that is less than $1,000 \mathrm{~Hz}$ to obtain the binaural beat. It should be noted that the difference between the two frequencies must be less than $30 \mathrm{~Hz}$ for the beating to be heard preferably around $440 \mathrm{~Hz}$. The binaural beats reportedly able to influence the brain through the entrainment of brainwaves and can be used to reduce anxiety and increase pain threshold [1]. These beats have recently gained interest and initially used by neurophysiologists and clinicians.

Previous studies have demonstrated that binaural beats can affect cortical responses across frequency bands. The most significant EEG steady-state responses occurred with a binaural beat of $40 \mathrm{~Hz}$ within the gamma band, and it primarily activated the frontal and parietal lobes [2]. Besides that, the EEG magnitude can be increased by $21 \%$ [3] when the binaural beat stimulation is in the beta band at $18.5 \mathrm{~Hz}$. The areas of cortex entrained by theta band binaural beats which included parietal, frontal, and temporal areas [4, 5]. However, binaural beats can be influenced by activity that is outside of the belonging frequency band which such effect is improperly characterized. A study by Gao et al. [ ] had shown that the EEG power increased in their respective band during either delta or alpha binaural beat stimulation and the theta and alpha bands [6]. The highest amount of synchronization can be obtained in the auditory cortex when the binaural beats occur within the beta band at $16 \mathrm{~Hz}$ [7].

Lane et al. [8] have improved the vigilance by exposing the binaural beats and the performance of participant is tested during a 1- back working memory test while listening to either theta or beta range binaural beats [59]. The improvement of target detection is seen from participants and the false alarms, task-related confusion, and fatigue are decreased while listening to binaural beats in the beta frequency range.

Besides that, Goodin [9] using different protocol by assessed theta $(7 \mathrm{~Hz})$ and beta $(16 \mathrm{~Hz})$ bands in 2-min $\mathrm{BB}$ carrier tone but the conclusion have differed from Lane et al. [8]. However, the author reported that the cortical frequency power during the period of BB stimulation is not significant differences as compared to a white noise signal. On the other hand, other positive effects of binaural beats have been reported on memory span task and verbal memory [10, 11]. These studies concluded that the binaural beat groups performed significantly better as compared to the control 
group, especially at a lower frequency. Another study reported the positive results of binaural beats in the experiment of relaxation [12], dual cognitive tasks [13], visuospatial working memory [14], pain and anxiety reduction [15-17], and severity ratings of tinnitus [18]. Other than that, another report indicates no changes occur on attention [19], blood pressure or heart rate variability (HRV) [20] and reduction on the symptoms of children diagnosed with attention deficit hyperactivity disorder (ADHD) [21].

The inconsistent studies-outcome as presented earlier is due to the methodological approaches, which include the following:

- Time duration of the experiment,

- Short tone vs continuous tone,

- Individual differences,

- Carrier frequency,

- Specific frequency to fluctuate the cortical activities,

- Attention to stimuli,

- Spatial limitation as the case of EEG,

- Age,

- Time of experiment; early morning or afternoon,

- Hearing ability, and

- Sensory modality.

\section{B. EDUCATION}

Education has many benefits beyond higher job status and salary. A higher education reduces the risks of substance abuse, crime and illnesses meanwhile improving the quality of life, social connectedness, and political participation. Positive feedback between performance on cognitive tests can be obtained such as IQ tests and scholastic achievement. The materials studied in school is known as "mental software" which are prepared for managing various cognitive domains such as: (1) mathematics, (2) reading, (3) writing and (4) understanding language, (5) problem solving in particular subject etc. Mental software would reduce one's mental load by clever encoding, organization, or processing [22].

Another form of learning known as "Wikipedia education" where its education is through teaching, research or trainer that formed by a group of people who are volunteer in transferring skill, habits and knowledge. Education can be any experience that has effects on the way of one thinks, feels or acts and it is commonly categorized into five stages in sequence which are starting from preschool, primary school, secondary school, college and university or apprenticeship $[23,24]$. As a consequence, education by default enhances cognitive abilities. However, effort may be required to improve the speed or efficiency of performance for a particular task special. Abacus, which is a simple mechanical calculation device that used to store numbers during mental calculations, which function to increase the speed of calculations; is an example of cognitive enhancement. In this device, the beads are slid up and down on various rods to simplify the mathematical processes such as subtraction, addition, division, multiplication, etc. unconventionally, a student is able to calculate without any physical abacus with frequent practice by their mentally picturing of beads. Surprisingly, many children trained on this technique are able to calculate faster than those who learnt with the help of electronic calculator.

\section{MENTAL TRAINING}

Mental training and visualization techniques are the commonly used practices in elite sport and rehabilitation, and it produces good effects on performance. Users imagine themselves vividly in performing a task, imagining repeatedly of every movement and the way of their felling. Mental training is a method to improve cognitive performance/ function using computerized and video gaming tasks [25-33]. Studies have demonstrated that computer gaming is able to improve significantly the cognitive control in older adults [33, 34]. The efficacy of such exercises can be likely explained by the activation of neural networks which involved in executing a skill simultaneously at a certain of time because the performance criteria for the task is justified by the close attention, optimizing neural plasticity and appropriate neural reorganization. General mental activity is training the brain muscle and that can improve the performance and long-term health, while relaxation techniques can help in regulating the activation of brain [35]. Similar studies were conducted to enhance the specific functions like visuomotor skills [36], working memory [37] and attention [38, 39].

Flynn-effect is the sustained and long-sustained increment of general cognitive abilities over the population with passage of time, and it has been suggested to produce a general increase in raw intelligence test scores by 2.5 IQ (intelligence quotient) points per ten years in most western countries [35]. Some other factors such as improved nutrition and health status that may increase the abstract and visual thinking [41] but the account of increased cognitive still the most demands by modern society and schooling.

Video gaming is another mental training technique in improving the cognitive abilities. Gaming is a good mental exercise because it could help a learner to have the better vision perception, attention and cognition by playing a fast paced game. Another example is playing the first person shooter game can improve the working memory [42]. Hence, video games are used in various fields such as education, physical exercise and cognitive training [37].

Another type of mental training consists of learning strategies to memorize information (i.e. memory techniques), one of such strategy is known as "The method of loci". Generally, this is a method of memory enhancement which employs visualization to organize and recall information. In this method, participant is immersed into a virtual reality view with the layout of shops on a street to postulate building or geographical entity in participant's imagination, so as to deposit the imaginary object while walking on the street from room to room. As a consequent, this method would evoke the natural associations to participant by the deposit memory. In addition, participant could recall also the memorized information while walking on the same street in the video training [35]. This method is normally used for tackling the spatial navigation system of brain to remember the objects or propositional contents.

\section{PHYSICAL EXERCISE}


Physical exercise is one of the methods to improve the executive performance and cognitive function. It is been demonstrated works by the evidence of aerobic physical activities that improve the cardiorespiratory fitness for the group of healthy and older adults, and with the beneficial effects for motor function, cognitive speed, delayed memory functions and auditory, and visual attention [43], The details can be found in [44].

Recent studies have shown that chronic exercise can lead to structural and functional changes in the brain and it can help in reducing the risks of cognitive decline and neurological diseases [45-51]. The non-exhaustive physical exercise has shown to be able to improve the activation of prefrontal cortex (PFC) through the increment of blood volume and its oxygenation level. Meanwhile, exercise is been generally known as an effective way of improving the executive performance in various tasks, such as decision-making and inhibitory control tasks [52, 53]. Among those tasks, the color-word matching Stroop task is the most current used technique in experimental and clinical settings in measuring the executive brain functions that localized in the prefrontal cortex [53-55]. Multiple studies have focused on the improvement of prefrontal activation due to benefits of physical exercise and its consequences on executive function. However, results can be differed depending on the task assessed during the bout of exercise, while some researchers [56] concluded the improvement in cognitive flexibility for shifting a broad attentional focus in the visual space through a computerized space and object-based attentional test, and others [57] found an impairment of the neuroelectric system which resulting in decreased task performance using the Flanker's task.

According to PFC, results might tend to suggest an improvement of cognitive capacities regardless of the age when the task is assessed after moderate exercise. For the pre-adolescent children, physical exercise has been associated with: (1) better performance on an academic achievement test, (2) a higher performance accuracy in the Flankers task, and (3) a larger P3 amplitude [58]. For young adults, a significant enhancement in left-Dorsolateral PFC and in left-Frontopolar Area (1-DLPFC and 1-FPA) has been observed and it is followed by the acute bouts of mild exercise and the combination of a shorter reaction time on the Stroop test [53]. For older adults, a similar effect has also been observed in the left-DLPFC [54] but another compensatory effect has also been noticed in the r-FPA [59]. Studies suggest that acute bouts of mild exercise are benefic in activating the PFC as well as the execution of tasks involving working memory.

\section{E. MEDITATION}

In recent years, meditation has become an increasingly popular as a topic of scientific research because of its suggested enhancing effects on brain and cognition. Several researches have indeed demonstrated that meditation practice, and in particular mindfulness meditation (MM), can promote changes in the brain structure and improvements in cognitive processing, such as executive functions and attention regulation abilities $[60,61]$. The practice of meditation has also been shown to lead to a variety of positive clinical outcomes, such as, for example, in anxiety, depression, stress, and substance abuse problems [62, 63]. Furthermore, more recently, meditation has been proposed to be a helpful method in delaying cognitive decline for elderly group and able to speed up the education of children to potentially reduce the risk of behavioral deviance and pathology [64].

Hence, meditation is one of the effective techniques for the improvement of cognitive abilities. From ancient period, meditation is practiced by humanity across the globe in some form or other. It is helpful to reduce anxiety, improve psychological health, reduce stress, improve memory, attention etc. Mindfulness meditation helps in reducing anxiety and pain [65] and it improves short term memory, attention and decreases reaction time [66]. Yoga is another technique used for enhancing cognitive abilities, that practice by alternanostril breathing pranayama (one of the oldest yoga) and it helps in reducing the mental stress, physical strength and improves health as well [67].

Rooks et al. [68] revealed the positive effects of shortform mindfulness training (MT) vs. relaxation training (RT) on sustaining the attention and emotional well-being for 100 college football players. Authors found that greater engagement in MT, but not RT, predicted greater benefits on the sustained attention to response task (SART). Such greater engagement in both training programs predicted negative change in anxiety and positive change in positive affect over a high-demand pre-season training interval. Jha et al. [69] investigated the possibility of mindfulness training (MT) in mitigating the working memory (WM) and also decreasing the susceptibility distraction that may be due to the persistent periods and intensive demands. Authors compared the effects on WM performance of two 8-weeks, 8hours variants of MT (versus a non-training group) in military cohorts for enduring a high-demand interval of military training and civilian cohort. Results showed that MT is variant that included of the in-class mindfulness training (vs. didactic content) was particularly effective in protecting the military cohorts against WM degradation over highdemand intervals.

Bennike et al. [70] compared the effects of two short-term (i.e., 30 days) online-based interventions in a randomized controlled design, focused on MM and brain training and behavioral (and indirect) markers of mind-wandering (using the SART) in two groups of healthy participants. Authors suggest that short online mindfulness-based interventions may be effective in reducing mind-wandering because they have observed that an increased ability in withholding a prepotent response during a monotonous task such as SART.

Hartkamp and Thornton [71] studied 41 individuals for a 6-day before and after intensive Vipassana (mindfulness) retreat and a series of behavioral measures assessing cognitive flexibility (e.g., Stroop, Task Switching) revealed no change in the retreat group vs. a group of control participants who did not take part to the retreat. Nevertheless, retreat participants reported increased mindfulness and well- being scores after the 6-day period. The limitations of this study gave a series of important suggestions for future studies on mindfulness and cognition. 
Böckler et al. [72] tested the effects of a 3-month contemplative training, which focused on observing-thought meditation and perspective taking of the healthy adult participants to practice the ability of their understanding as well as others' mental states. Result shows that the degree of selfunderstanding is improved and meanwhile understand others' mental states as well, this condition is known as the theory of mind (TOM) performance. Besides that, Kohler et al. [73] investigated the effects of a concentrative form of yoga nidra meditation on attention performance for a sample of ten young healthy participants. The participants are not allowing to sleep before and after the test and also after a session of meditation or rest.

Eventually, it is observed that the sustained attention is improved following by meditation and the sleepiness is decreased. This finding indicate a role for meditation in improving the deficits attention that included of sleep loss. According to brain imaging studies, Simon et al. [74] reported that the concentration can be increased, and the deactivations can be induced within the default mode network (DMN) by a specific practice of mantra meditation. This investigation bring a new finding to this specific area as the previous research studies was only focus on mantra meditation for the activations within DMN. Meanwhile, authors Authors found that a 2-week period of Kundalini yoga/meditation can decreased the activations within subregions of the DMN. This is showing that the training in mantra meditation such as focused attention and open monitoring are having a suppressive effect on activity within the DMN. In another brain imaging study, Kozasa et al. [75] investigated a difference in DMN connectivity between regular meditators and nonmeditators during an attention Stroop word-color paradigm. The finding reveals that the connectivity between the precuneus/posterior cingulate cortex and the right and left parietal cortices helps in differentiating the regular meditators from non-meditators, suggesting that the two populations have different connectivity patterns in the DMN reflecting different degree of interference in attention processes. Mooneyham et. al. [76] revealed that a 6-week mindfulness-based health and wellness intervention led to a significant increase in cortical thickness in the left posterior insula and to a significant increased functional connectivity of this area with the right ventrolateral prefrontal cortex and with the left middle and superior temporal gyri, areas which are related to interoception and attention mechanisms.

Last, Sato et al. [77] investigated the structural and functional brain changes in a single meditator before and after a 5.5- week retreat. The findings are observed after the retreat of decreased in regional homogeneity in the precuneus and decreased of cortical thickness in visual cortices, Brodmann area 8 and anterior cingulate, together with increased amplitude of low-frequency fluctuations in dorsolateral prefrontal cortex, were tentatively held to reflect the development of a focused and calm mind and decreased mind-wandering.

\section{F. TRANSCRANIAL DIRECT CURRENT SIMULATION}

transcranial direct current stimulation (tDCS) has been a technique to improve cognition [78, 79] in recent years. tDCS is a non-invasive technique that function by passing a direct electrical current through the cerebral cortex via electrodes placed upon the scalp. The current is of low amplitude and below the threshold for potential induction and it leads to changes in neuronal excitability through membrane polarization and changes in synaptic strength [80]. This technique can be used to change the excitability of neurons in a polarity-dependent manner, such that spontaneous neural activity within a specific region can be either increased or decreased depending on the placement of electrode (i.e. the anode or positive electrode or the cathode or negative electrode). The changes in cortical excitability which can lasted more than 1 hour have been demonstrated [81] after a few minutes of stimulation. For healthy adults, a single session of tDCS using small currents (1-2 mA) has been shown to transiently improve 'online' (i.e. during the period of stimulation) cognitive performance on executive tasks including verbal fluency [82-84], WM tasks [85, 86], attention [87], language [88], problem solving[89] and measures of learning and memory [90]. Another study [91] reported that tDCS was applied to the parietal lobes of healthy adults during training sessions with artificial numerical characters, and the stimulation of these brain areas resulted in long-lasting enhancement of numerical proficiency.

Similarly, a recent tRNS study shows that concurrent stimulation of the parietal lobes could boost long-term task performance with discernable effects up to 16 weeks [92] during the training of numerosity discrimination task .

Researches indicated that tDCs improves the vigilance [98] [99] and enhanced the performance on majority of CT tasks[93]. This technique have a positive impact on improving the cognitive function of people with cognitive impairments [95], depression [96] and that with old adults with functional limitations [97]. Additionally, studies have shown the effectiveness of tDCS as a method to alleviate vigilance problems at work [100]. The following figure shows the tDCs simulations sites.

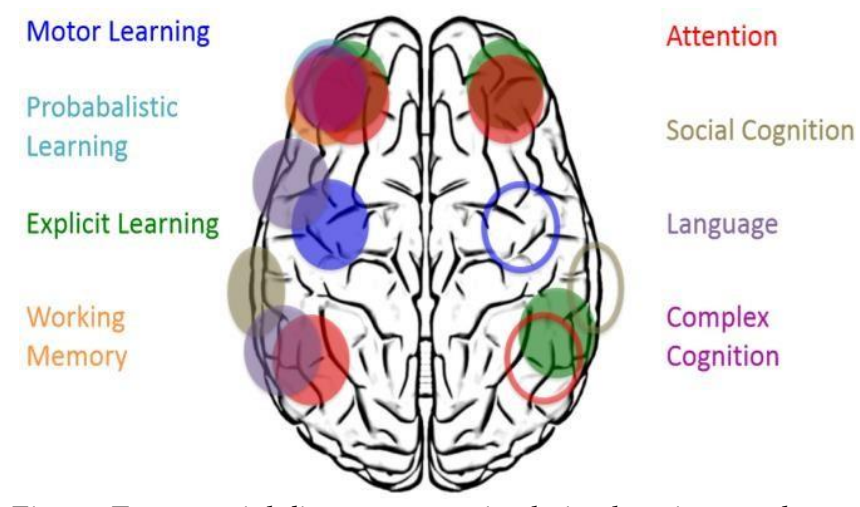

Figure .Transcranial direct current stimulation locations on the brain.

\section{G.DRUGS}

The general perception of drugs is used for enhancing cognitive abilities, but these are illegal and unhealthy. However, some drugs are legal, and they are very useful for cognitive enhancement when taken in proper amount. Cognitive abilities like memory, attention, and concentration can be improved by drugs such as nootropics. Nootropics work by improving the brain's oxygen supply, and stimulating nerve growth, or by altering the availability 
of the brain's supply of neurochemicals (neurotransmitters, enzymes, and hormones). Nootropics formulations have been used in ayurvedic system since ages. Some examples for enhancing memory are almonds, ginger, tulsi, ambla, Indian tulip tree etc [39].

\section{H. NEUROFEEDBACK}

Neurofeedback is a method that assists subjects to control their brain waves consciously by recording the electroencephalography (EEG) during the neurofeedback treatment. Its various components are extracted and fed to subjects using online feedback loop in the form of audio, video or their combination while the electrophysiological components are separately demonstrated. The power of a signal in a frequency band can be shown by a varying bar graph to assess participants progress on achieving the optimum performance. For instance, the subject tries to improve the brain patterns based on the changes that occur in the sound or movie. Neurofeedback treatment protocols mainly focus on the alpha, beta, delta, theta, and gamma treatment or a combination of them such as alpha/theta ratio, beta/theta ratio, etc., [101]. However, the most commonly used protocols are alpha, beta, theta, and alpha/theta ratio, detail can be found in [102].

6 types of Neurofeedback were reported for the disorder's treatment:

Firstly, the most frequently used neurofeedback is frequency/power neurofeedback. This technique typically uses 2 to 4 surface electrodes and it is sometimes called "surface neurofeedback". This method is used to change the amplitude or speed of a specific brain waves in particular brain locations to treat ADHD, anxiety, and insomnia. The second method is known as slow cortical potential neurofeedback (SCP-NF) that is used to improve the direction of slow cortical potentials to treat ADHD, epilepsy, and migraines [103]. The third method is "Low-energy neurofeedback system (LENS)" which it is function by delivering a weak electromagnetic signal to change the patient's brain waves in a condition where participant must be motionless, and both eyes are closed [104]. This type of neurofeedback has been used to treat traumatic brain injury, ADHD, insomnia, fibromyalgia, restless legs syndrome, anxiety, depression, and anger. The fourth method is "Hemoencephalographic (HEG) neurofeedback". It provides feedback on cerebral blood flow to treat migraine [105] and the Live Z-score neurofeedback is used to treat insomnia. To provide continuous feedback, it introduces the continuous comparison of variables of brain electrical activity to a systematic database [106]. The fifth method is known as "Low-resolution electromagnetic tomography (LORE-TA)". It functions by using the 19 electrodes to monitor phase, power, and coherence [107]. This neurofeedback technique is used to treat addictions, depression, and obsessive-compulsive disorder. The sixth method is the Functional magnetic resonance imaging (fMRI) which is the most recent type of neurofeedback method to regulate brain activity based on the activity feedback from deep subcortical areas of the rain [108].

\section{MUSIC}

Music has been identified as a useful stimulus for various healing and rehabilitation practices [109]. Earlier studies showed that a short time exposure to music is able to enhance the spatio-temporal performance of brain regions, this is known as popularly known as the Mozart effect [110]. However, subsequent findings claimed that the effect is not limited to Mozart's composition or to spatio-temporal reasoning. Further studies proposed that any stimulus that induces a moderate level of arousal and pleasant mood in the subject is able to result in a significant enhancement in the cognitive performances. This effect disappears when arousal and pleasantness are held constant and it can be referred to arousalmood hypothesis which states that the enhanced cognitive performances are effects of stimulus on the mood of the subject while no music induced direct activation of neurons to enhance cognitive performances.

A comprehensive review by Schellenberg et al. concluded that music listening improves the cognitive performance, albeit, only in the short term. Results from a recent review has emphasized that music listening has many inherent benefits for cognitive, motor, emotional, and social functioning. On the other hand, few earlier studies supported the claim that music listening is directly influencing the brain networks for enhancing spatial abilities. Another study showed that music activates brain regions responsible for attention and cognitive tasks. A recent finding suggests that music activates regions which are linked to memory, cognition, and IQ. However, the previous studies were not able to convincingly establish the detailed and systematically parameterize of the direct mechanisms between music and cognitive enhancement. Few recent works reveal a new aspect of interconnections between music and brain signals such as EEG and fMRI are both similar in being the scale free of nature. According to musical perspective, sonification of brain signals into music has given a deeper insight for neuro-activities of brain. Recent study have demonstrated that short-term exposure to music enhanced global efficiency of the brain, prefrontal efficiency and increased sustained attention [111].

\section{J. CAFFEINE}

Caffeine is often in the form of coffee or tea and it has been a popular material to enhance various aspects of mental or cognitive functions. Certain details regarding specific functional aspects remain controversial although there is widespread scientific agreement about caffeine's behavioral effects. For instance, there is general consensus that caffeine improves "lower" cognitive functions such as simple reaction time, whereas caffeine's effects on "higher" cognitive functions such as problem solving, and decision making are often debated because only a fewer published studies on higher-order cognitive function, and those publications are greatly varying with respect to the employed methods.

Caffeine increases arousal in a dose-dependent manner; low doses can improve hedonic tone and may reduce anxiety, while high doses increase tension and symptoms of anxiety, nervousness and jitteriness. The caffeine in doses from 32 to $300 \mathrm{mg}$ is a scientific standard that able to enhance the fundamental aspects of cognitive performance, such as attention, vigilance, and reaction time [112]. However, low and moderate doses of caffeine have not been shown to alter the sensory functions (i.e., vision or hearing) to a significant degree. The effect of caffeine on performance is depending on the part of individuals arousal level who is under 
investigation, especially for the extent of which subjects are sleep-deprived or fatigued versus well- rested.

According to [113-116], the extent to which caffeine either improves or degrades sensory function can be partially explained by the classic inverted U- shaped arousal performance function. According to the Yerkes-Dodson law, there is an empirical relationship between arousal and performance, such that low arousal is associated with poor performance whereas increased physiological or mental arousal is associated with improved performance, but it's limited at a specific point.

The observed effects of pre-dose arousal level is influenced by the consumed caffeine whereas the performance decreases when arousal level becomes too high.

Administration of a large dose of caffeine to an individual who is severely fatigued likely will improve performance because in this case, caffeine promotes a favorable arousal level (i.e., caffeine advances the subject's arousal into the middle range of the Yerkes-Dodson curve). Conversely, performance may degrade rather than improve by giving the same dose to someone who already is well-rested and highly aroused because caffeine produces a state of overarousal and degrade cognition, according to Yerkes-Dodson law. Caffeine is employed to achieve a self-perceived, peak state of arousal in day-to-day circumstances by selecting an optimal level of arousal and cognitive performance (in accordance with the Yerkes-Dodson law). Studies reveal that the doses under investigation are properly matched to the individual's arousal state, and eventually the beneficial effects are likely to be observed.

\section{REFERENCES}

[1] J. C. R. Licklider, J. Webster, and J. J. T. J. o. t. A. S. o. A. Hedlun, "On the frequency limits of binaural beats," vol. 22, no. 4, pp. 468-473, 1950.

[2] R. Draganova, B. Ross, A. Wollbrink, and C. J. C. c. Pantev,

"Cortical steady-state responses to central and peripheral auditory beats," vol. 18, no. 5, pp. 1193-1200, 2007.

[3] J. A. Frederick, J. F. Lubar, H. W. Rasey, S. A. Brim, and J. J.

J. o. N. Blackburn, "Effects of $18.5 \mathrm{~Hz}$ auditory and visual stimulation on EEG amplitude at the vertex," vol. 3, no. 3-4, pp. 23-28, 1999.

[4] R. J. J. o. N. Kennerly, "QEEG analysis of binaural beat audio entrainment: A pilot study," vol. 8, pp. 122-122, 2004.

[5] H. Pratt, A. Starr, H. J. Michalewski, A. Dimitrijevic, N.

Bleich, and N. J. C. n. Mittelman, "Cortical evoked potentials to an auditory illusion: binaural beats," vol. 120, no. 8, pp.

$1514-1524,2009$

[6] X. Gao et al., "Analysis of EEG activity in response to binaural beats with different frequencies," vol. 94, no. 3, pp. 399406 ,

2014.

[7] D. C. Fitzpatrick, J. M. Roberts, S. Kuwada, D. O. Kim, and B.

J. J. o. t. A. f. R. i. O. Filipovic, "Processing temporal modulations in binaural and monaural auditory stimuli by neurons in the inferior colliculus and auditory cortex," vol. 10, no. 4, p. 579, 2009.

[8] J. D. Lane, S. J. Kasian, J. E. Owens, G. R. J. P. Marsh, and behavior, "Binaural auditory beats affect vigilance performance and mood," vol. 63, no. 2, pp. 249-252, 1998.

[9] P. Goodin, J. Ciorciari, K. Baker, A.-M. Carrey, M. Harper, and J. J. P. o. Kaufman, "A high-density EEG investigation into steady state binaural beat stimulation," vol. 7, no. 4, p. e34789,
[10] A. Fernández, F. Maestu, P. Campo, R. Hornero, J. Escudero, and J. J. A. E. P. Poch, "Impact of auditory stimulation at a frequency of $5 \mathrm{~Hz}$ in verbal memory," vol. 36, no. 6, pp. 307-

313, 2008.

[11] R. C. J. W. G. C. Kennerly, Carrolton, GA, "An Empirical Investigation Into the Effect of Beta Frequency Binaural-beat Audio Signals on Four Measures of Human Memory,

\section{ADD/ADHD," 1994}

[12] P. A. McConnell, B. Froeliger, E. L. Garland, J. C. Ives, and G.

A. J. F. i. p. Sforzo, "Auditory driving of the autonomic nervous system: Listening to theta-frequency binaural beats postexercise increases parasympathetic activation and sympathetic withdrawal," vol. 5, p. 1248, 2014.

[13] B. Hommel, R. Sellaro, R. Fischer, S. Borg, and L. S. J. F. i. p.

Colzato, "High-frequency binaural beats increase cognitive flexibility: evidence from dual-task crosstalk," vol. 7, p. 1287,

2016

[14] C. Beauchene, N. Abaid, R. Moran, R. A. Diana, and A. J. P. o.

Leonessa, "The effect of binaural beats on visuospatial working memory and cortical connectivity," vol. 11, no. 11, p. e0166630, 2016.

[15] K. Ecsy, A. K. P. Jones, and C. A. J. E. J. o. P. Brown, "Alpha-range visual and auditory stimulation reduces the perception of pain," vol. 21, no. 3, pp. 562-572, 2017.

[16] T. J. Weiland et al., "Original sound compositions reduce anxiety in emergency department patients: a randomised controlled trial," vol. 195, no. 11-12, pp. 694-8, 2011.

[17] D. Wiwatwongwana, P. Vichitvejpaisal, L. Thaikruea, J.

Klaphajone, A. Tantong, and A. J. E. Wiwatwongwana, "The effect of music with and without binaural beat audio on operative anxiety in patients undergoing cataract surgery: a randomized controlled trial," vol. 30, no. 11, p. 1407, 2016.

[18] J. B. David, A. Naftali, and A. J. T. H. J. Katz, "Tinntrain: a multifactorial treatment for tinnitus using binaural beats," vol.

63, no. 11, pp. 25-26, 2010.

[19] A. Crespo, M. Recuero, G. Galvez, and A. J. A. o. A. Begona, "Effect of binaural stimulation on attention and EEG," vol. 38, no. 4, pp. 517-528, 2013.

[20] C. J. J. o. h. m. Carter and p. relations, "Healthcare performance and the effects of the binaural beats on human blood pressure and heart rate," vol. 18, no. 2, pp. 213-219,

2008.

[21] S. Kennel, A. G. Taylor, D. Lyon, and C. J. J. o. p. n.

Bourguignon, "Pilot feasibility study of binaural auditory beats for reducing symptoms of inattention in children and adolescents with attention-deficit/hyperactivity disorder," vol.

25 , no. 1, pp. 3-11, 2010.

[22] A. Sandberg and N. J. E. E. P. Bostrom, "Cognitive enhancement: a review of technology," 2006.

[23] H. Kirschenbaum, 100 Ways To Enhance Values and Morality in Schools and Youth Settings. ERIC, 1995.

[25] A. Lampit, H. Hallock, and M. J. P. m. Valenzuela,

"Computerized cognitive training in cognitively healthy older adults: a systematic review and meta-analysis of effect modifiers," vol. 11, no. 11, p. e1001756, 2014.

[26] A. M. Kueider, J. M. Parisi, A. L. Gross, and G. W. J. P. o.

Rebok, "Computerized cognitive training with older adults: a systematic review," vol. 7, no. 7, p. e40588, 2012.

[27] A. J. Jak, A. M. Seelye, and S. M. J. N. r. Jurick, "Crosswords to computers: a critical review of popular approaches to cognitive enhancement," vol. 23, no. 1, pp. 13-26, 2013.

[28] A. M. Owen et al., "Putting brain training to the test," vol. 465, no. 7299 , p. 775, 2010.

[29] L. Djabelkhir et al., "Computerized cognitive stimulation and engagement programs in older adults with mild cognitive impairment: comparing feasibility, acceptability, and cognitive and psychosocial effects," vol. 12, p. 1967, 2017.

[30] P. L. Baniqued et al., "Cognitive training with casual video games: points to consider," vol. 4, p. 1010, 2014.

[31] A. C. Oei and M. D. J. P. O. Patterson, "Enhancing cognition with video games: a multiple game training study," vol. 8 , no. 
3 , p. e58546, 2013

[32] A. C. Oei and M. D. J. F. i. s. n. Patterson, "Are videogame training gains specific or general?," vol. 8, p. 54, 2014.

[33] J. A. Anguera et al., "Video game training enhances cognitive control in older adults," vol. 501, no. 7465, p. 97, 2013

[35] N. Bostrom, A. J. S. Sandberg, and e. ethics, "Cognitive enhancement: methods, ethics, regulatory challenges," vol. 15, no. 3, pp. 311-341, 2009.

[36] S. A. Houthoofd, M. Morrens, and B. G. J. C. t. Sabbe, "Cognitive and psychomotor effects of risperidone in schizophrenia and schizoaffective disorder," vol. 30, no. 9, pp.

1565-1589, 2008

[37] Al-Shargie, Fares, Masashi Kiguchi, Nasreen Badruddin, Sarat C. Dass, Ahmad Fadzil Mohammad Hani, and Tong Boon Tang. "Mental stress assessment using simultaneous measurement of EEG and fNIRS." Biomedical optics express 7, no. 10 (2016): 3882-3898.

[38] Al-Shargie, F. M., Tong Boon Tang, Nasreen Badruddin, and Masashi Kiguchi. "Mental stress quantification using EEG signals." In International Conference for Innovation in Biomedical Engineering and Life Sciences, pp. 15-19. Springer, Singapore, 2015.

[39] Al-Shargie, Fares, Tong Boon Tang, and Masashi Kiguchi. "Assessment of mental stress effects on prefrontal cortical activities using canonical correlation analysis: an fNIRS-EEG study." Biomedical optics express 8, no. 5 (2017): 2583-2598.

[40] Al-Shargie, Fares, Tong Boon Tang, and Masashi Kiguchi. "Stress assessment based on decision fusion of EEG and fNIRS signals." IEEE Access 5 (2017): 19889-19896.

[41] Al-Shargie, Fares, Tong Boon Tang, Nasreen Badruddin, and Masashi Kiguchi. "Towards multilevel mental stress assessment using SVM with ECOC: an EEG approach." Medical \& biological engineering \& computing 56, no. 1 (2018): 125-136.

[42] Al-shargie, Fares, Tong Boon Tang, and Masashi Kiguchi. "Mental stress grading based on fNIRS signals." In 2016 38th Annual International Conference of the IEEE Engineering in Medicine and Biology Society (EMBC), pp. 5140-5143. IEEE, 2016.

[43] Al-shargie, Fares, Tong Boon Tang, Nasreen Badruddin, and Masashi Kiguchi. "Simultaneous measurement of EEG-fNIRS in classifying and localizing brain activation to mental stress." In 2015 IEEE International Conference on Signal and Image Processing Applications (ICSIPA), pp. 282-286. IEEE, 2015.

[44] Al-Shargie, Fares. "Early Detection of Mental Stress Using Advanced Neuroimaging and Artificial Intelligence." arXiv preprint arXiv:1903.08511 (2019).

[45] Mohamed, Eltaf Abdalsalam, Mohd Zuki Yusoff, Ibrahim Khalil Adam, Elnazeer Ali Hamid, Fares Al-Shargie, and Muhammad Muzammel. "Enhancing EEG Signals in Brain Computer Interface Using Intrinsic Time-Scale Decomposition." In Journal of Physics: Conference Series, vol. 1123, no. 1, p. 012004. IOP Publishing, 2018

[46] Al-shargie, Fares, Tong Boon Tang, Nasreen Badruddin, Sarat C. Dass, and Masashi Kiguchi. "Mental stress assessment based on feature level fusion of fNIRS and EEG signals." In 2016 6th International Conference on Intelligent and Advanced Systems (ICIAS), pp. 1-5. IEEE, 2016.

[47] Al-Shargie, Fares. "Multilevel Assessment of Mental Stress using SVM with ECOC: An EEG Approach." (2019).

[48] Al-Yoonus, Murthad, M. F. L. Abdullah, Mohammed Saeed Jawad, and Fares Al-Shargie. "Enhance quality control management for sensitive industrial products using 2D/3D image processing algorithms." In 2014 Electrical Power, Electronics, Communicatons, Control and Informatics Seminar (EECCIS), pp. 126-131. IEEE, 2014.

[48] Al-Shargie, Fares. "Assessment of Mental Stress Using EEG and fNIRS Features." (2019).

[49] Jawad, Mohammed Saeed, Fares Al-Shargie, Murthad AlYoonus, and Zahriladha bin Zakaria. "Performance Analysis Comparative Study of Fingerprint Recognition Systems." Computers and Software (2014): 1154.
[49] Al-shargie, Fares, Tong Boon Tang, Nasreen Badruddin, and Sarat C. Dass. "Prefrontal cortex connectivity under neutralcontrol and stress condition using fNIRS."

[50] Al-Shargie, Fares. "Quantification of Mental Stress using fNIRS Signals." (2019).

[51] Al-Shargie, Fares. "Fusion of fNIRS and EEG Signals: Mental Stress Study." (2019).

[51] Amalarethinam, DI George, P. Muthulakshmi, Mohammed Saeed Jawad, Fares Al-Shargie, Murthad Al-Yoonus, Zahriladha bin Zakaria, Sampath Prakasam et al. "Computers and Software." (2014).

[51] Omar, Nazlia, and Qasem Al-Tashi. "Arabic nested noun compound extraction based on linguistic features and statistical measures." GEMA Online® Journal of Language Studies 18, no. 2 (2018).

[52] Al-Tashi, Qasem, Said Jadid Abdul Kadir, Helmi Md Rais, Seyedali Mirjalili, and Hitham Alhussian. "Binary Optimization Using Hybrid Grey Wolf Optimization for Feature Selection." IEEE Access 7 (2019): 39496-39508.

[53] Al-Tashi, Qasem Abdullah Qasem. "The Development of ECensus of Population System." PhD diss., Universiti Teknologi Malaysia, 2012.

[54] Al-Tashi, Qasem. "WORD SENSE DISAMBIGUATION: A REVIEW." (2019).

[51] Al-Tashi, Qasem. "Feature Selection." (2019).

[50] Al-Tashi, Qasem, Helmi Rais, and Said Jadid. "Feature Selection Method Based on Grey Wolf Optimization for Coronary Artery Disease Classification." In International Conference of Reliable Information and Communication Technology, pp. 257-266. Springer, Cham, 2018.

[50] Al-Tashi, Qasem, Helmi Rais, and Said Jadid Abdulkadir. "Hybrid Swarm Intelligence Algorithms with Ensemble Machine Learning for Medical Diagnosis." In 2018 4th International Conference on Computer and Information Sciences (ICCOINS), pp. 1-6. IEEE, 2018.

[51] E. Bullitt et al., "The effect of exercise on the cerebral vasculature of healthy aged subjects as visualized by MR angiography," vol. 30, no. 10, pp. 1857-1863, 2009.

[52] L. F. Ciria, P. Perakakis, A. Luque-Casado, and D. J. N.

Sanabria, "Physical exercise increases overall brain oscillatory activity but does not influence inhibitory control in young adults," vol. 181, pp. 203-210, 2018.

[53] K. Byun et al., "Positive effect of acute mild exercise on executive function via arousal-related prefrontal activations: an fNIRS study," vol. 98, pp. 336-345, 2014.

[54] K. Hyodo et al., "The association between aerobic fitness and cognitive function in older men mediated by frontal lateralization," vol. 125, pp. 291-300, 2016.

[55] S. Kujach et al., "A transferable high-intensity intermittent exercise improves executive performance in association with dorsolateral prefrontal activation in young adults," vol. 169, pp.

117-125, 2018.

[56] C. Pesce, L. Capranica, A. Tessitore, and F. J. J. o. H. M. $\mathrm{S}$.

Figura, "Effects of a sub-maximal physical load on the orienting and focusing of visual attention," vol. 42, no. 5, pp. 401-420, 2002.

[57] M. B. Pontifex and C. H. J. C. N. Hillman, "Neuroelectric and behavioral indices of interference control during acute cycling," vol. 118, no. 3, pp. 570-580, 2007.

[58] C. H. Hillman, M. B. Pontifex, L. B. Raine, D. M. Castelli, E.

E. Hall, and A. F. J. N. Kramer, "The effect of acute treadmill walking on cognitive control and academic achievement in preadolescent children," vol. 159, no. 3, pp. 1044-1054, 2009.

[59] K. Hyodo et al., "Acute moderate exercise enhances compensatory brain activation in older adults," vol. 33, no. 11 , pp. 2621-2632, 2012.

[60] Y.-Y. Tang, B. K. Hölzel, and M. I. J. N. R. N. Posner, "The neuroscience of mindfulness meditation," vol. 16, no. 4, p. 213, 
[61] K. C. Fox et al., "Is meditation associated with altered brain structure? A systematic review and meta-analysis of morphometric neuroimaging in meditation practitioners," vol. 43, pp. 48-73, 2014.

[62] A. Chiesa, R. Calati, and A. J. C. p. r. Serretti, "Does mindfulness training improve cognitive abilities? A systematic 464, 2011. review of neuropsychological findings," vol. 31, no. 3, pp. 449

[63] S. G. Hofmann, A. T. Sawyer, A. A. Witt, D. J. J. o. c. Oh, and c. psychology, "The effect of mindfulness-based therapy on anxiety and depression: A meta-analytic review," vol. 78, no. 2, p. $169,2010$.

[64] T. Gard, B. K. Hölzel, and S. W. J. A. o. t. N. Y. A. o. S. Lazar, "The potential effects of meditation on age-related cognitive decline: a systematic review," vol. 1307, no. 1, pp. 89-103, 2014.

[65] J. J. Miller, K. Fletcher, and J. J. G. h. p. Kabat-Zinn, "Threeyear follow-up and clinical implications of a mindfulness meditation-based stress reduction intervention in the treatment of anxiety disorders," vol. 17, no. 3, pp. 192-200, 1995.

[67] B. S. Oken et al., "Randomized, controlled, six-month trial of yoga in healthy seniors: effects on cognition and quality of life," vol. 12, no. 1, p. 40, 2006.

[68] J. D. Rooks, A. B. Morrison, M. Goolsarran, S. L. Rogers, and A. P. J. J. o. C. E. Jha, "WWe Are Talking About Practice": the Influence of Mindfulness vs. Relaxation Training on Athletes' Attention and Well-Being over High-Demand Intervals," vol. 1, no. 2, pp. 141153,2017

[69] A. P. Jha, J. E. Witkin, A. B. Morrison, N. Rostrup, and E. J. J. o. C. E. Stanley, "Short-form mindfulness training protects against working memory degradation over high-demand intervals," vol. 1, no. 2, pp. 154-171, 2017.

[70] I. H. Bennike, A. Wieghorst, and U. J. J. o. C. E. Kirk, "Onlinebased mindfulness training reduces behavioral markers of mind wandering," vol. 1, no. 2, pp. 172-181, 2017.

[72] A. Böckler, L. Herrmann, F.-M. Trautwein, T. Holmes, and T.

J. J. o. C. E. Singer, "Know thy selves: Learning to understand oneself increases the ability to understand others," vol. 1, no. 2, pp. 197209,2017

[76] B. W. Mooneyham, M. D. Mrazek, A. J. Mrazek, K. L.

Mrazek, E. D. Ihm, and J. W. J. J. o. C. E. Schooler, "An integrated assessment of changes in brain structure and function of the insula resulting from an intensive mindfulness-based

intervention," vol. 1, no. 3, pp. 327-336, 2017.

[77] J. R. Sato, E. H. Kozasa, B. A. Wallace, and E. J. J. o. C. E. Amaro, "Neuroimaging data from a single participant before and after a meditation retreat: a proof of concept study," vol. 1, no. 2, pp. 235-241, 2017.

[82] M. Iyer, U. Mattu, J. Grafman, M. Lomarev, S. Sato, and E. J. N. Wassermann, "Safety and cognitive effect of frontal DC brain polarization in healthy individuals," vol. 64, no. 5, pp. 872-875, 2005.

[83] R. Hurley, L. J. J. o. c. Machado, and e. neuropsychology, "Using transcranial direct current stimulation to improve verbal working memory: A detailed review of the methodology," vol.

40, no. 8, pp. 790-804, 2018.

[84] V. S. d. S. dos Santos et al., "Cognitive effects of transcranial direct current stimulation combined with working memory training in fibromyalgia: a randomized clinical trial," vol. 8, no.

1, p. 12477,2018

[85] T. Zaehle, P. Sandmann, J. D. Thorne, L. Jäncke, and C. S. J.

B. n. Herrmann, "Transcranial direct current stimulation of the prefrontal cortex modulates working memory performance: combined behavioural and electrophysiological evidence," vol

12 , no. 1, p. $2,2011$.

[86] S. P. Ruf, A. J. Fallgatter, and C. J. S. R. Plewnia, "Augmentation of working memory training by transcranial direct current stimulation (tDCS)," vol. 7, no. 1, p. 876, 2017.

[87] B. A. Coffman, V. P. Clark, and R. J. N. Parasuraman, "Battery

powered thought: enhancement of attention, learning, and memory in healthy adults using transcranial direct current stimulation," vol. 85 , pp. 895-908, 2014
[88] R. Sparing, M. Dafotakis, I. G. Meister, N.

Thirugnanasambandam, and G. R. J. N. Fink, "Enhancing language performance with non-invasive brain stimulation-a transcranial direct current stimulation study in healthy humans," vol. 46, no. 1, pp. 261-268, 2008.

[89] C. Cerruti and G. J. J. o. c. n. Schlaug, "Anodal transcranial direct current stimulation of the prefrontal cortex enhances complex verbal associative thought," vol. 21, no. 10, pp. 1980-

$1987,2009$.

[90] A. H. Javadi and V. J. B. s. Walsh, "Transcranial direct current stimulation (tDCS) of the left dorsolateral prefrontal cortex modulates declarative memory," vol. 5, no. 3, pp. 231-241,

2012.

[91] R. K. Cohen, S. Soskic, T. Iuculano, R. Kanai, and V. J. C. b.

C. Walsh, "Modulating neuronal activity produces specific and long-lasting changes in numerical competence," vol. 20, no. 22, pp. 2016 2020, 2010.

[92] J. W. Schuijer, I. M. de Jong, F. Kupper, and N. M. J. F. i. h. n. van Atteveldt, "Transcranial Electrical Stimulation to Enhance Cognitive Performance of Healthy Minors: A Complex Governance Challenge," vol. 11, p. 142, 2017.

[93] J. Elmasry, C. Loo, D. J. R. n. Martin, and neuroscience, "A

systematic review of transcranial electrical stimulation combined with cognitive training," vol. 33, no. 3, pp. 263-278,

2015.

[94] B. A. Simonsmeier, R. H. Grabner, J. Hein, U. Krenz, M. J. N.

Schneider, and B. Reviews, "Electrical brain stimulation (tES)

improves learning more than performance: a meta-analysis,"

2017.

[95] P. Cruz Gonzalez, K. N. Fong, and T. J. B. n. Brown, "The Effects of Transcranial Direct Current Stimulation on the Cognitive Functions in Older Adults with Mild Cognitive Impairment: A Pilot Study," vol. 2018, 2018.

[96] M. A. Nitsche, P. S. Boggio, F. Fregni, and A. J. E. n. PascualLeone, "Treatment of depression with transcranial direct current stimulation (tDCS): a review," vol. 219, no. 1, pp. 14-

$19,2009$.

[97] B. Manor et al., "Transcranial Direct Current Stimulation May Improve Cognitive-Motor Function in Functionally Limited Older Adults," vol. 32, no. 9, pp. 788-798, 2018.

[98] J. T. Nelson, R. A. McKinley, E. J. Golob, J. S. Warm, and R.

J. N. Parasuraman, "Enhancing vigilance in operators with prefrontal cortex transcranial direct current stimulation (tDCS)," vol. 85, pp. 909-917, 2014.

[99] R. A. McKinley, N. Bridges, C. M. Walters, and J. J. N.

Nelson, "Modulating the brain at work using noninvasive transcranial stimulation," vol. 59, no. 1, pp. 129-137, 2012.

[100] R. K. Mehta and R. J. F. i. h. n. Parasuraman,

"Neuroergonomics: a review of applications to physical and cognitive work," vol. 7, p. 889, 2013.

[101] D. J. J. A. p. Vernon and biofeedback, "Can neurofeedback training enhance performance? An evaluation of the evidence with implications for future research," vol. 30, no. 4, p. 347,

2005

[102] H. Marzbani, H. R. Marateb, M. J. B. Mansourian, and c. neuroscience, "Neurofeedback: a comprehensive review on system design, methodology and clinical applications," vol. 7 no. 2, p. 143, 2016.

[103] H. Christiansen, V. Reh, M. H. Schmidt, and W. J. F. i. h. n.

Rief, "Slow cortical potential neurofeedback and self-management training in outpatient care for children with ADHD: study protocol and first preliminary results of a

randomized controlled trial," vol. 8, p. 943, 2014.

[104] Y. Zandi Mehran, M. Firoozabadi, R. J. C. E. Rostami, and neuroscience, "Improvement of neurofeedback therapy for improved attention through facilitation of brain activity using local sinusoidal extremely low frequency magnetic field exposure," vol. 46, no. 2, pp. 100-112, 2015.

[105] Á. M. Dias, A. M. Van Deusen, E. Oda, and M. R. J. T. S. j. o. 
p. Bonfim, "Clinical Efficacy of a New Automated Hemoencefalographic Neurofeedback Protocol," vol. 15, no. 3, pp. 930-941, 2012.

[106] T. F. Collura, J. Guan, J. Tarrant, J. Bailey, and F. J. J. o. N.

Starr, "EEG biofeedback case studies using live Z-score training and a normative database," vol. 14, no. 1, pp. 22-46,

2010 .

[107] R. D. Pascual-Marqui, C. M. Michel, and D. J. I. J. o. p.

Lehmann, "Low resolution electromagnetic tomography: a new method for localizing electrical activity in the brain," vol. 18, no. 1, pp. 49$65,1994$.

[109] T. J. A. o. p. Särkämö and r. medicine, "Cognitive, emotional, and neural benefits of musical leisure activities in aging and neurological rehabilitation: A critical review," 2017.

[110] F. H. Rauscher, G. L. Shaw, and K. N. J. N. 1. Ky, "Listening to Mozart enhances spatial-temporal reasoning: towards a neurophysiological basis," vol. 185, no. 1, pp. 44-47, 1995.

[111] A. Gupta, B. Bhushan, and L. J. S. r. Behera, "Short-term enhancement of cognitive functions and music: A three-channel model," vol. 8 , no. 1 , p. 15528,201

[112] . Porciúncula, L.; Ardais, A.; Espinosa, J.; Mioranzza, S.; Rocha, A.; Sallaberry, C.J.J.o.N. the Janus face of caffeine: s04-02. Journal of Neurochemistry 2013, 125, 17.

[113]. Kamimori, G.H.; Johnson, D.; Thorne, D.; Belenky, G.J.A., space, environmental medicine. Multiple caffeine doses maintain vigilance during early morning operations. Aviation, Space, Environmental Medicine 2005, 76, 1046-1050.

[114]. Frewer, L.; Lader, M.J.H.P.C.E. The effects of caffeine on two computerized tests of attention and vigilance. Human

Psychopharmacology: Clinical Experimental 1991, 6, 119-128.

[115]. Lane, J.D.; Phillips-Bute, B.G.J.P.b. Caffeine deprivation affects vigilance performance and mood. Physiology Behavior 1998, 65, $171-175$.

[116]. Lieberman, H.; Wurtman, R.; Emde, G.; Roberts, C.; Coviella, I.J.P. The effects of low doses of caffeine on human performance and mood. Psychopharmacology 1987, 92, 308-312. 